Klaus-Dieter Bernert

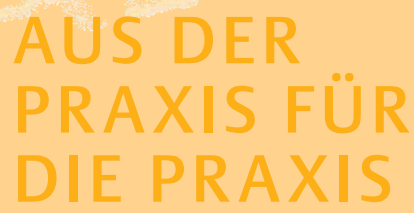

\section{Vorbemerkungen}

Zunächst ein paar Bemerkungen dazu, wie wir zur Neuraltherapie kamen. Der Weg vom Medizinstudium über die ersten Praktika bis hin zur Facharztausbildung und der klassischen Schulmedizin, der wir alle sehr viel zu verdanken haben, ist spannend.

Dann kommt ein sog. „ausdiagnostizierter“ und „austherapierter“ Patient in die Praxis. Diese Situation ist furchtbar unangenehm, denn der Patient berichtet, dass keiner etwas gefunden habe und er u.a. von Schmerzmitteln und Psychopharmaka lebe - irgendwie scheint es keinen Ausweg zu geben.

Hier kommt die Neuraltherapie nach Huneke mit ihrem Störfeldgedanken und der Verknüpfung des vegetativen Nerven-

\section{Kasten 1 \\ Praxisbeispiel}

Eine junge Frau hatte vor einem Jahr eine Sectio und schläft seitdem nicht mehr durch. Die Ursache könnte das unruhige Kind sein, aber die Suche blieb ergebnislos. Jetzt kommt die ganzheitliche Verknüpfung unserer Denkweise hinzu: Die Sectionarbe könnte der störende Faktor sein nichts ist leichter, als diese Narbe mit Procain zu unterspritzen.

Die Patientin berichtet wenige Tage nach der Injektion: „Ich habe das erste Mal nach einem Jahr wieder geschlafen und bin Ihnen so dankbar!“

\title{
Neuraltherapie nach Huneke
}

\section{Praxiserprobte Konzepte}

\section{Zusammenfassung}

Als moderne Regulationstherapie stellt die Neuraltherapie das Bindeglied zwischen Naturheilkunde und Schulmedizin dar. Die Begründer der Neuraltherapie - die Brüder Ferdinand und Walter Huneke - prägten schon sehr früh den Begriff des Störfelds, bei dem es auch zur Fernwirkung auf andere Bereiche und Organe kommen kann. Der Beitrag zeigt den breiten Behandlungsspielraum der Methode.

\section{Schlüsselwörter}

Neuraltherapie, Huneke, Störfeld, gynäkologischer Raum.

\section{Abstract}

As a modern regulatory therapy, neural therapy is the connecting link between naturopathy and orthodox medicine. The founders of neural therapy - the brothers Ferdinand and Walter Huneke - very early coined the term interference field. This term means, that even a remote effect on other areas and organs is possible. The article shows the ample scope of treatment of this method.

\section{Keywords}

Neural therapy, Huneke, neuralgias, neurological diseases, interference field, gynecological space.

systems ins Spiel. Die Suche nach der Ursache der Beschwerden ist ein kriminalistisches Vorgehen: Wann und wo ist im Körper eine Unruhe entstanden, die die Selbstheilungskräfte versagen lässt? Ein Beispiel aus dem Praxisalltag (Kasten 1) soll dies verdeutlichen.

\section{Kurzer geschichtlicher Abriss}

Die Begründer der Neuraltherapie nach Huneke sind die Brüder Ferdinand und Walter Huneke, die im Jahre 1928 in ihrer Veröffentlichung „Unbekannte Fernwirkung der Lokalanästhesie" Einblicke in ihre Arbeit zuließen [4, 5]. Zuvor waren es schon einige, die die therapeutische Anwendung von Lokalanästhetika beschrie- ben haben. Es begann mit Einhorn im Jahre 1905, dem die Novocainsynthese gelang. Dann sind z.B. 1894 Schleich, 1902 Spieß und 1920 Leriche zu nennen. Ebenfalls im Jahre 1928 berichteten Leriche und Fontane über „schnellere Wundheilung an Frakturstellen durch Lokalanästhesie“ [4, 7]. 1940 veröffentlichte Ferdinand Huneke „Das Sekundenphänomen“ [6].

1964 erschien das „Lehrbuch der Neuraltherapie nach Huneke“ von Peter Dosch [2] und 1991 wurde die Ute-Huneke-Stiftung gegründet, die die Neuraltherapie in der Schmerzambulanz der Heinrich-Heine-Universität Düsseldorf etablierte.

Weitere Bücher und Atlanten folgten, z.B. „Lehrbuch und Atlas der Neuraltherapie nach Huneke“ von Hans Barop [1], „Neuraltherapie nach Huneke: Neurophysiologie, 


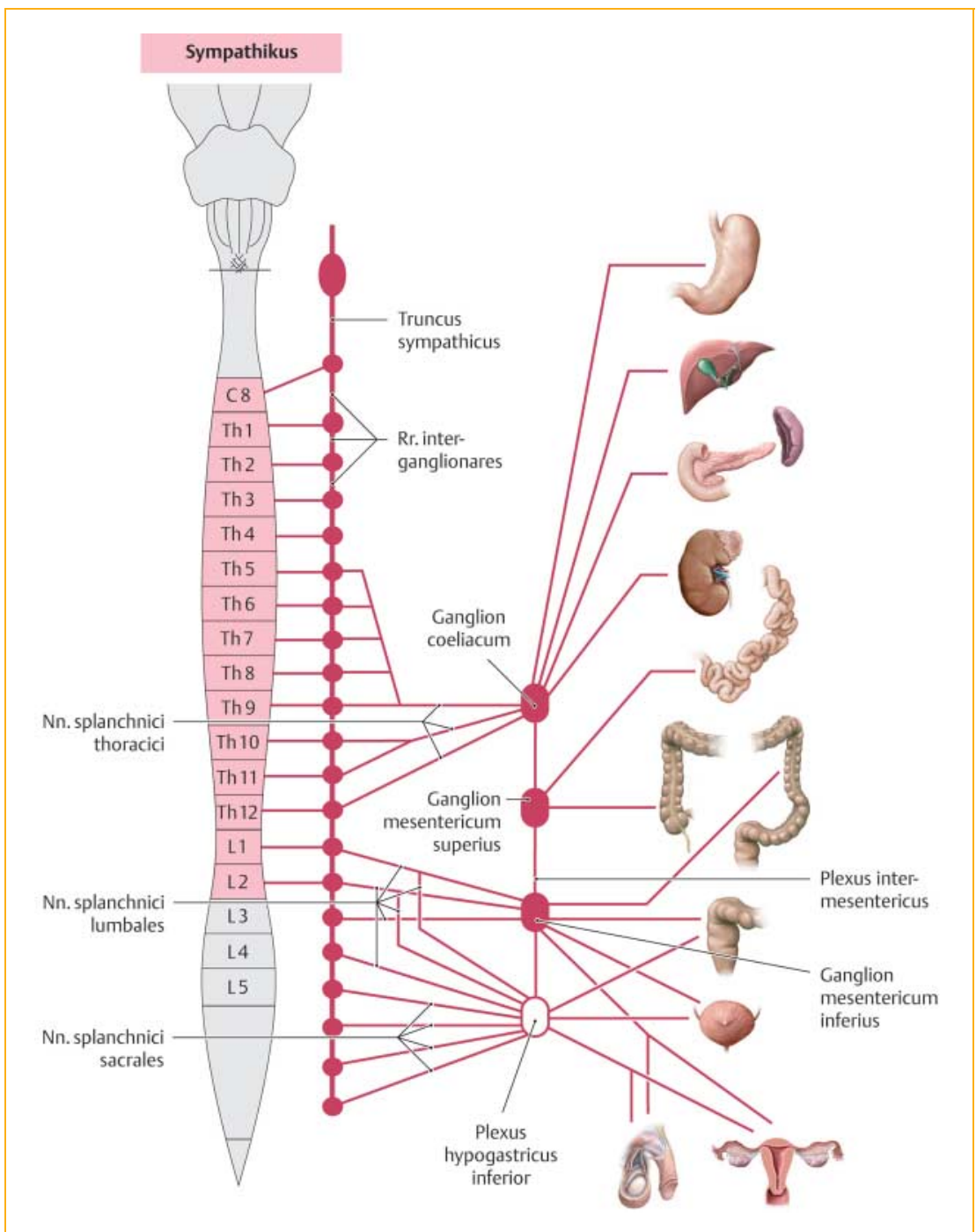

Abb.1: Schematische Darstellung des Sympathikus und seiner Vernetzung. @ Schünke M, Schulte E, Schumacher U: Prometheus. LernAtlas der Anatomie. Stuttgart: Thieme; 2009. Illustration: M. Voll

Injektionstechnik und Therapievorschläge“ von Lorenz Fischer [3], „Handbuch Neuraltherapie: Diagnostik und Therapie mit Lokalanästhetika“ von Stefan Weinschenk [8].

\section{Was kann die Neural- therapie?}

- Anregung körpereigener Heilungskräfte

- Behandlung von akuten und chronischen Erkrankungen

- Therapie im Segment (da, wo es wehtut)

- Ausschaltung sog. Störfelder, die Fernwirkung haben

\section{Durchführung der Neural- therapie}

Zum Spritzen wird fast ausschließlich das Lokalanästhetikum Procain $1 \%$ ohne Konservierungsstoffe (!) verwendet (5 mlAmpullen). Man kann Procain aber auch systemisch als Infusion verwenden (Procain-Basen-Infusion).

Die Neuraltherapie ist eine moderne Regulationstherapie. Sie ist eine der bekanntesten Naturheilverfahren und stellt das Bindeglied zwischen Naturheilkunde und Schulmedizin dar. Anatomische Grundlage ist das Grundregulationssystem, das modifiziert nach Pischinger und Heine bekannt ist. Über dieses Grundregulationssystem können alle therapeutischen Verfahren zur Anwendung kommen, deren Konzept in der Wiederherstellung der körpereigenen Regulationsmechanismen besteht (z.B. auch bei der Homöopathie oder Akupunktur).

Dass dies alles so gut funktioniert ist im Prinzip nur dadurch zu erklären, dass das vegetative Nervensystem ubiquitär verteilt ist. Eine herausragende Rolle spielt hier der Sympathikus, der durch seine hochgradige Vernetzung sämtlicher Organe diese Regulation ermöglicht (Abb.1).

Aufgrund sehr genauer Beobachtungen durch die Gebrüder Huneke kam der Begriff des Störfelds sehr früh zur Anwendung. Was damals schon postuliert wurde, sehen wir heute auch so, denn jede chronische Erkrankung kann störfeldbedingt sein. Jede Erkrankung oder Verletzung kann ein Störfeld hinterlassen und jede Störfelderkrankung ist nur durch „Ausschaltung“ des Störfelds heilbar.

Wie immer beginnt in der Medizin alles mit einer ausführlichen Krankengeschichte, und zwar von Geburt an bis jetzt. Es folgt eine gründliche Untersuchung, ggf. kommen alle z.Z. möglichen Diagnoseverfahren zur Anwendung. Diese allerdings erst nach gründlicher Überlegung und Abwägung.

Typische Störfelder sind:

- die Tonsillen,

- die Nasennebenhöhlen,

- der Zahn- und Kieferbereich,

- Prostata und gynäkologischer Raum sowie

- Narben aller Art (der Nabel ist die erste Narbe, die alle haben!).

Die Neuraltherapie nach Huneke hat eine sehr breite Fächerung; im wahrsten Sinne des Wortes von Kopf bis Fuß. Dieses moderne Regulationsverfahren mit Einfluss auf alle Regelkreise des Organismus ist dadurch gekennzeichnet, dass es am nervalen, hormonellen, muskulären, zirkulatorischen und lymphatischen System reguliert und Einfluss auf Skelett, Verdauungs- und Ausscheidungsorgane hat.

\section{Was ist im Einzelnen therapier- bar?}

Kopf: Kopfschmerzen, Migräne, Kopfdruck, Folgen von Gehirnerschütterungen und Schädelbrüchen wie Schwindel, 
Gleichgewichtsstörungen oder Epilepsie als Unfallfolge. Trigeminusneuralgien, Fazialislähmungen

Augen: Glaukom und alle entzündlichen Augenerkrankungen wie Neuritis, Iridozyklitis, Keratitis, Skleritis

Ohren: akute und chronische Mittelohrentzündung, vom Ohr ausgehende Gleichgewichtsstörungen (Menière-Krankheit), Ohrensausen und Ohrgeräusche, Schwerhörigkeit

Nase: Symptome und Diagnosen wie Heuschnupfen, Ozeana, chronischer Schnupfen, Verlust des Geruchsvermögens, Nasennebenhöhleneiterungen

Hals: Schilddrüsenvergrößerungen mit und ohne Über- oder Unterfunktion, Nervosität, chronische Mandelentzündung, ständiges Druck- und Fremdkörpergefühl, Peitschenschlagsyndrom nach Auffahrunfällen

Brust: Angina pectoris, Herzstiche, Herzbeklemmung, Zustand nach Herzinfarkt, nervöse Beschwerden ohne erkennbare Ursache

Bauchbereich: Leber- und Gallenleiden, Zwölffingerdarm-Geschwüre, Erkrankung der Bauchspeicheldrüse, chronische Verstopfung, chronischer Durchfall, Kolitis

Unterleib der Frau: Entzündungen der Gebärmutter, Eileiter und Eierstöcke, Periodenschmerzen, Ausfluss, Krankheiten, die nach Fehlgeburten oder schweren Entbindungen aufgetreten sind, Kinderlosigkeit, sexuelle Störungen, Schwangerschaftserbrechen, Neigung zu Fehlgeburten, Bettnässen. Neu: Erkrankungen, die durch Papillomaviren verursacht werden (HPV).

Mann: Veränderungen an der Prostata wie Vergrößerung, Entzündungen, damit einhergehende Impotenz, Nierenerkrankungen, Blasenleiden wie Reizblase und Bettnässen

Gelenke: alle möglichen Formen der Schmerzen bei Arthrosis deformans (Altersund Abnutzungserkrankungen mit Zackenbildung)

Wirbelsäule: Zervikalsyndrom, Spondylose, Osteoporose, Bandscheibenschaden, Bechterew-Krankheit, Koxarthrose, Hexenschuss, Kniebeschwerden, Gelenkund Muskelrheuma, Arthritis, Steißbeinschmerzen, Bänderzerrungen, Muskelrisse und deren Folgen
Außerdem: Tennisellenbogen, Überanstrengungen anderer Art nach Unfällen, z.B. Schmerzen am Amputationsstumpf, organische Durchblutungsstörungen an Armen und Beinen, Sudeck-Syndrom, Gefäßkrämpfe, Lymphabflussstauungen, Sportverletzungen

Haut: chronische Hautleiden wie Ekzeme, aber v.a. Narbenschmerzen, Keloidnarben, Entzündungen aller Art, Afterund Scheidenjuckreiz, Hämorrhoidenbeschwerden, Thrombosen, Schmerzen nach Gürtelrose, Warzen, Furunkel, schlecht heilende Wunden, offene Beine

Nerven: Neuralgien aller Art, Nervenentzündungen, Ischias, Gemütsveränderungen nach Krankheiten oder Operationen, „nervöse Organleiden“, Gefühlsstörungen, funktionelle Erkrankungen, Schmerzzustände aller Art, Polyneuropathien

Gefäße: arterielle und venöse Durchblutungsstörungen, Krampfadern, Unterschenkelgeschwüre, Alters- und Abnutzungserkrankungen, postoperative Zustände, Störungen der Hormondrüsen, aber auch Wetterfühligkeit und Fönkrankheit

\section{Wo ist Zurückhaltung geboten?}

Bei Krankheiten wie Schizophrenie, manisch-depressivem Syndrom und Hysterie ist nach wie vor der Psychiater gefragt.

Bei seelisch bedingten Krankheiten und Gemütsbewegungen wie Angst, Sorgen oder Schreck, die zu bleibenden seelischen Gleichgewichtsstörungen geführt haben, bedarf es des Psychotherapeuten. In letzter Zeit kristallisiert sich allerdings heraus, dass auch bei depressiven Verstimmungen und den geschilderten Gemütslagen Injektionen durchaus sinnvoll sind; z.B. an das Ganglion supremum. Sehr häufig kann so der Medikamentenbedarf deutlich reduziert bis aufgehoben werden!

Alle Mangelzustände müssen natürlich ausgeglichen werden.

Erbkrankheiten kann man ebenfalls nicht mit der Neuraltherapie behandeln.

Krebserkrankungen sind selbstverständlich nicht durch Procain heilbar, aber Linderungen der Schmerzen sind durchaus möglich und zusätzlich zur gesamten Schulmedizin sinnvoll. Die Beseiti- 
gung von Störfeldern schafft dann häufig Linderung und führt zur Besserung des Befindens.

Da dieser Artikel v.a. Neuralgien und neurologische Erkrankungen beleuchten soll, beschränken sich die folgenden Ausführungen im Wesentlichen darauf.

\section{Wie wird im Einzelfall vorgegangen?}

Je nach Grunderkrankung werden die Injektionen dort vorgenommen, wo das Problem steckt, es werden also z.B. Narben unterspritzt. Eine einzige Injektion kann z.B. dazu führen, dass Kopfschmerzen dauerhaft verschwinden (Sekundenphänomen).

In meiner Praxis „landen“ häufig Frauen mit regelmäßig wiederkehrenden Schmerzen während der Menstruation, sowohl als Dysmenorrhö als auch mit Kopfschmerzen in dieser Zeit.

\section{Vorgehen bei Kopfschmerzen:}

Zunächst Quaddeln im HWS-Bereich und Injektionen an die Triggerpunkte im Halsund Schulterbereich setzen, ggf. Quaddeln über den Austrittspunkten der Trigeminusäste (Foramina supraorbitalis, infraorbitalis, selten mentalis, jeweils bds.).

Sehr gut helfen auch der Kopfkranz nach Hopfer und die Behandlung der Triggerpunkte am Kopf, meist über dem Os temporale. Hier verwende ich Procain $1 \%$. Bringt diese Behandlung nur vorübergehend Linderung, gilt es, an das Störfeld zu denken. Sehr häufig ist das der gynäkologische Raum.

\section{Vorgehen bei menstruell bedingten Schmerzen:}

- Quaddeln von Th 8-L 2 paravertebral

- Quaddeln und Injektion an die Sakralnerven von S 2-S 4

- Injektion an die Schilddrüse $(0,5 \mathrm{ml}$ Procain $1 \%), 1 \mathrm{ml}$ i.v., zusätzlich ein wenig perivasal

- Alle Narben im Segment werden unterspritzt (Sectio-, Appendektomie-, Herniotomie- und Laparoskopienarben, aber auch Narben nach Exstirpation von Hauttumoren).

- Der Nabel wird unterspritzt.
Als letztes gehört noch der eigentliche gynäkologische Raum dazu. Hier gibt es 2 Möglichkeiten:

- der abdominale Zugang zum Frankenhäuser Plexus,

- der vaginale Zugang.

Beide haben ihre Berechtigung, da man an unterschiedliche Strukturen herankommt.

Mit diesem Vorgehen habe ich die besten Erfolge erzielt.

In seltenen Fällen kommen auch andere Störfelder in Betracht. In diesem Fall nehme ich Testinjektionen an die Stellen vor, die aufgrund der Anamnese und der Untersuchung als Störfeld infrage kommen. Zum anderen denke ich aufgrund der Häufigkeiten der Störfelder immer an den Kopf-, Zahn- und Kieferbereich und spritze an die entsprechenden Zähne und das Ganglion pterygopalatinum bds.

\section{Weitere Behandlungsmöglichkeiten:}

Lässt sich damit keine Besserung erzielen, versuche ich - dem ganzheitlichen Gedanken weiter folgend - mögliche Mangelzustände zu beheben:

- Vitaminmangel

- Mineralstoffmangel

- Hormonmangel

Eine weitere, sehr etablierte Methode, die auch bei anderen Schmerzzuständen z.B. bei Polyneuropathien oder Fibromyalgien - zur Anwendung kommt, ist die Procain-Basen-Infusion.

Mit dem aufgeführten Konzept kann ich in sehr vielen Fällen eine deutliche Linderung der Beschwerden herbeiführen, häufig verschwinden diese sogar völlig.

Bei anderen Schmerzen, wie Rückenschmerzen (vom Hals bis zum Steißbein), beginne ich immer damit, direkt am Ort des Schmerzes zu quaddeln, Triggerpunkte zu beheben und Myogelosen zu spritzen.

\section{Noch ein Hinweis zur Methode}

Selbstverständlich kann jeder approbierte Arzt Quaddeln im Segment durchführen und Narben unterspritzen. Aber tiefe Injektionen, z.B. an den gynäkologischen bzw. andrologischen Raum und an Ganglien - und hier möchte ich besonders das Ganglion cervicale superius, das Gang- lion stellatum und das Ganglion coeliacum hervorheben - sollten wirklich nur von ausgebildeten Neuraltherapeuten durchgeführt werden. Informationen zur Ausbildung sind z.B. unter www.ignh.de zu finden.

Interessenkonflikte: Der Autor erklärt, dass keine wirtschaftlichen oder persönlichen Verbindungen bestehen.

\section{Online zu finden unter:}

http://dx.doi.org//10.1055/s-0033-1357571

\section{Literatur}

[1] Barop H. Lehrbuch und Atlas der Neuraltherapie nach Huneke. Stuttgart: Hippokrates; 1996

[2] Dosch P. Lehrbuch der Neuraltherapie nach Huneke. 14. Aufl. Heidelberg: Haug; 1995

[3] Fischer L. Neuraltherapie nach Huneke. Neurophysiologie, Injektionstechnik und Therapievorschläge. 3. Aufl. Stuttgart: Hippokrates; 2007

[4] Fontaine R. Neurochirurgie des vegetativen Nervensystems. In: Monnier M, Hrsg. Physiologie und Pathophysiologie. Stuttgart: Hippokrates; 1963: 832-883

[5] Huneke F, Huneke W. Unbekannte Fernwirkung der Lokalanästhesie. Med Welt 1928; 27: 1013-1014

[6] Huneke F. Das Sekunden-Phänomen in der Neuraltherapie - mit einem Nachtrag von Hagen Huneke. 6. Aufl. Heidelberg: Haug; 1989

[7] Leriche R. Chirurgie des Schmerzes. Leipzig: Johann Ambrosius Barth; 1958. Übersetzung von La Chirurgie de la douleur. 3. Aufl. Paris: Masson; 1949

[8] Weinschenk S, Hrsg. Handbuch Neuraltherapie: Diagnostik und Therapie mit Lokalanästhetika. München: Elsevier; 2010

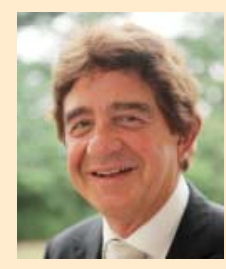

Klaus-Dieter Bernert studierte Medizin in Jena und ist Facharzt für Gynäkologie und Geburtshilfe. 1990

Niederlassung als Frauenarzt in eigener Praxis in Usingen und Belegarzt am dortigen Krankenhaus. Seit 1993 fachübergreifende Gemeinschaftspraxis mit seiner Ehefrau. Zertifizierter Neuraltherapeut nach Huneke mit Tätigkeit als Tutor.

Korrespondenzadresse

Dr. med. Klaus-Dieter Bernert

Franz-Schubert-Str. 2-4

61250 Usingen

E-Mail: dr.bernert@gmx.de 